Archives

$10 \mid 1993$

Le Centre de recherches historiques de 1949 à 1975

\title{
Le démarrage improvisé : 1949-1956
}

\section{(2) OpenEdition}

Journals

\section{Édition électronique}

URL : http://journals.openedition.org/ccrh/2788

DOI : $10.4000 /$ ccrh. 2788

ISSN : $1760-7906$

Éditeur

Centre de recherches historiques - EHESS

Édition imprimée

Date de publication : 15 avril 1993

ISSN : 0990-9141

Référence électronique

"Le démarrage improvisé : 1949-1956 », Les Cahiers du Centre de Recherches Historiques [En ligne],

10 | 1993, mis en ligne le 16 mars 2009, consulté le 01 mai 2019. URL : http://

journals.openedition.org/ccrh/2788; DOI : 10.4000/ccrh.2788

Ce document a été généré automatiquement le 1 mai 2019.

Article L.111-1 du Code de la propriété intellectuelle. 


\section{Le démarrage improvisé : 1949-1956}

1 En janvier 1949, le Centre de recherches historiques commençait avec des moyens plus que modestes. Installé dans des locaux loués par le Centre de politique étrangère, il s'agissait d'un petit secrétariat animé par V. Chomel, archiviste-paléographe, assisté de deux secrétaires dactylographes. Les activités scientifiques étaient le fait de deux chercheurs du CNRS, Desaunay et Trimbach, et de jeunes chercheurs comme R. Romano, arrivé à Paris en décembre 1947 et depuis mars 1948 en contact avec Fernand Braudel et "tout le monde des Annales ${ }^{1}$ », (P. Chaunu, J. Ebersolt ${ }^{2}$ ou M. Duchaillut, future Mme Baulant). Le nouveau «laboratoire de recherches historiques» consacra ses activités scientifiques au rassemblement et au dépouillement de documents d'histoire économique des seizième et dix septième siècles. Deux types de document ont retenu la curiosité et les efforts des chercheurs du Centre tout au long des années 1950, des correspondances de marchands dont ils préparèrent l'édition scientifique ${ }^{3}$ et des séries longues de prix et de quantités de marchandises témoignant des mouvements du commerce européen ${ }^{4}$.

2 La création d'un nombre croissant de postes au CNRS permit au directeur du nouveau centre, F. Braudel, d'engager des stagiaires et attachés de recherche tenus d'investir au moins 54 jours de travail à un «but d'intérêt collectif» dans des projets qui exigeaient surtout des compétences archivistiques. En 1952-53, ils étaient déjà 10 : Mme Salmi, MM. Soboul, Dautry, Blumenkranz, Baehrel, Goubert, Godinho, de Prada, Angel, Tenenti, Bertin et Chomel ${ }^{5}$. À cette liste, le rapport ajoute les noms d'autres chercheurs, de formation historique ou archivistique, rémunérés par le CNRS : Mmes Baulant, Guillemin, Gardenat, Hirsch, Helmrich et MM. da Silva et Somella. Marc Bouloiseau, élève de G. Lefebvre, attaché de recherche au CNRS et responsable de l'édition des discours de Robespierre, projet commun de la Société des études robespierristes, du CNRS et du CRH, succéda au premier secrétaire en 1951 et coordonna les activités multiples du Centre jusqu'en 1955. Mais l'inspiration intellectuelle de son directeur, Fernand Braudel, sur des collaborateurs encore très jeunes, était le véritable lien de coordination dans cette phase de démarrage improvisé. Sa thèse publiée en 1949 livrait à la fois le cadre thématique et la problématique des recherches individuelles. Le programme de l'équipe réunie autour de lui était la description concrète et complète des structures et conjonctures économiques du seizième et du dix septième siècle. Ce programme, qui incitait à résoudre les problèmes 
de dépouillement des sources à la fois massives et disparates de l'histoire économique, trouva un premier écho dans le groupe croissant de disciples d'E. Labrousse qui animait les recherches d'histoire économique et sociale à la Sorbonne. Le CRH de ces premières années fait penser à un atelier de dimensions modestes voyant passer toute une série de compagnons qui rendent visite à un maître et s'en repartent avec des concepts et des programmes de travail nouveaux.

Pendant ces années, le CRH concentra ses ressources de travail collectif à l'édition. Il s'engagea dans une activité ambitieuse de publication: d'abord, les trois collections devenues classiques - "Affaires et Gens d'affaires", "Ports - Routes - Trafics» et "Monnaie - Prix - conjoncture »- balisaient les terrains de recherche que le jeune Centre voulait s'approprier et fournissaient en même temps les premiers éléments d'une documentation scientifique sur "les rythmes et les courants de la vie matérielle de $l^{\prime} E u r o p e ~ d u \mathrm{XV}^{e}$ au XVIII ${ }^{e}$ siècle $^{6} »$. L'édition des lettres de marchands, des premières séries de prix et de volumes de marchandises du commerce naval et routier aux $\mathrm{XVI}^{\mathrm{e}}$ et $\mathrm{XVII}^{\mathrm{e}}$ siècle européen, avait souvent «le caractère d'une édition de matériaux semi-élaborés » qui livrait une masse énorme de faits nouveaux pour l'histoire économique et sociale de ces époques? ${ }^{7}$.

4 Ces activités d'édition et de publication absorbèrent une part toujours croissante des moyens financiers et humains du $\mathrm{CRH}^{8}$. En 1955, $53 \%$ des dépenses du CRH étaient consacrées à ce secteur - pourcentage qui ne tient pas compte de la somme, inconnue, allouée aux vacations accordées aux travaux d'édition'. En fait, le CRH était au centre d'une politique offensive de publication qui correspondait aux intentions des directeurs de la $\mathrm{VI}^{\mathrm{e}}$ Section de marquer publiquement sa force d'innovation et son dynamisme. Un texte de la présidence adressé aux responsables de l'enseignement supérieur en 1954 exprime bien cette stratégie. Sous le titre triomphant «En moins de trois ans, la VIe Section a publié » il donne la liste de 39 « ouvrages originaux d'histoire économique et sociale, d'ethnographie, d'économie » dont 19 relèvent de l'histoire ${ }^{10}$. D'une part, la bibliothèque générale de la $\mathrm{VI}^{\mathrm{e}}$ Section présentait les premières activités de la nouvelle institution et de ses professeurs ${ }^{11}$ et servait, ensemble avec les Cahiers des Annales, à diffuser les nouveaux textes « classiques » de « l'école des Annales ${ }^{12}$ ». D'autre part, le CRH s'engageait, au delà de ses éditions de sources d'histoire économique, dans la traduction $d^{\prime}$ 'ouvrages étrangers ${ }^{13}$ et la publication de thèses d'histoire économique et sociale ${ }^{14}$.

Par cette politique, le CRH devenait un pôle d'attraction pour tous les chercheurs en histoire économique et sociale en quête d'éditeur de sorte que son directeur se trouvait confronté à un nombre toujours croissant de manuscrits. Dans sa nouvelle fonction de secrétaire du CRH, succédant à M. Bouloiseau, D. Ozanam avertit F. Braudel, fin 1957, des risques qu'une telle politique éditoriale présentait pour le Centre. Le nombre de manuscrits acceptés pour publication s'était élevé à une soixantaine et formait un stock pour au moins trois ans au rythme actuel de 20 livres par an. « Il est hors de doute qu'en continuant dans cette voie le Centre court le péril de manquer à sa vocation de recherche pour devenir une véritable maison d'édition ${ }^{15}$. » Mais ce n'est qu'en 1960 que Braudel fut prêt à accepter les conseils de prudence et à freiner l'expansion des activités éditoriales qui risquaient de compromettre l'originalité du programme scientifique du Centre ${ }^{16}$. Le tableau 5 permet de voir la dynamique des années 1950 et le ralentissement décidé au début des années 1960 . 
Tableau 5. Nombre de volumes publiés dans les collections du CRH (1951-1962)

\begin{tabular}{|c|c|c|c|c|c|c|c|}
\hline Nom de la collection & $\begin{array}{l}1951 \\
1952\end{array}$ & $\begin{array}{l}1.953 \\
1.954\end{array}$ & $\begin{array}{l}1955 \\
1956\end{array}$ & $\begin{array}{l}195 \% \\
1958\end{array}$ & $\begin{array}{l}1.959 \\
1.960\end{array}$ & $\begin{array}{l}1961 \\
1952\end{array}$ & "Foutaill \\
\hline Forts-Roules-"Traftics & 4 & 1 & 8 & 3 & 8 & 2 & 24 \\
\hline Affures el Gens dafiares & 1 & 6 & 2 & 3 & 10 & 6 & 28 \\
\hline Prix.Monale-Conjoncture & it & (1) & 3 & (1) & 1 & 1. & 6 \\
\hline Ouvages étrangers & - & 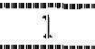 & 1 & 1 & - & -.. & 3 \\
\hline I.usp hom & ....... & - & 1 & 1 & 3 & and & ? \\
\hline $\begin{array}{l}\text { Documents at recherchess sult } \\
\text { l'écon des peys byzantins }\end{array}$ & 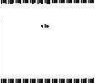 & - & $=$ & 1 & 2 & 2 & 5 \\
\hline Dérnographie el Sociés & -. & - & $=$ & 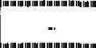 & 5 & 3 & 8 \\
\hline 4:Uldes & 2 & 1 & - & - & l & $\because$ & 4 \\
\hline Tolat & 8 & 9 & 13 & 9 & 30 & 16 & 85 \\
\hline
\end{tabular}

Les chiffres indiquent le nombre de volumes pour tenir compte des titres publiés en plusieurs tomes. La rubrique «Autres » comprend les trois volumes des discours de Robespierre et un de la collection "Livre et Société ».

6 Comparées à ses programmes de publication, les autres activités du CRH furent beaucoup plus modestes.

7 Les quatre enquêtes du tableau 6 présentent un bilan plutôt maigre des premières tentatives de recherche collective au $\mathrm{CRH}$. Seules les deux premières aboutirent à des publications, celles sur les mouvements de prix et sur la démographie sont restées inachevées.

Tableau 6. Enquêtes collectives du CRH de 1949 à 1959

\begin{tabular}{|c|c|c|}
\hline $\begin{array}{l}1949 a \\
1954\end{array}$ & $\begin{array}{l}\text { Les discours de Robespierre ; dépouille- } \\
\text { ment de la presse révolutionnaire. }\end{array}$ & $\begin{array}{l}\text { Bouloisea (dir.), Dautry, } \\
\text { Soboul, Salmi-Bianchi }\end{array}$ \\
\hline 1949.50 & Lepéage de Jougne et les routes transjuranes & Chomel, Ebersollt \\
\hline $\begin{array}{l}1951 \\
a \\
1954-5\end{array}$ & $\begin{array}{l}\text { Mouvements des prix à Parils aux XVIe et } \\
\text { XVules; depouillement des archives de l'as- } \\
\text { sistance publique. }\end{array}$ & $\begin{array}{l}\text { Goubert,Baehurel, Sommellat } \\
\text { Helmurch. Gardénat; B aulant } \\
\text { Guillemin. }\end{array}$ \\
\hline $\begin{array}{l}1953 \\
a \\
1959\end{array}$ & $\begin{array}{l}\text { Démographie de l'Arncien Régirne : dépouil- } \\
\text { lement des registres paroissiaux-; définition } \\
\text { d'une struoture démographique de type an- } \\
\text { cien et des cycles dénographiques }\end{array}$ & $\begin{array}{l}\text { Goubert (dir.), Baehrel; Ré- } \\
\text { mond, Bouloiseanu; vaca-- } \\
\text { taires. }\end{array}$ \\
\hline
\end{tabular}

Sources : budgets de la VIe Section, rapports d'activité du CRH 1952/53; rapport à la réunion du CRH du 19 décembre 1957. Pour qu'une enquête soit considérée comme collective, elle doit engager au moins deux chercheurs simultanément. Le sort de la dernière enquête, sur la démographie de l'Ancien Régime, reste énigmatique : après l'avoir préparée, Baehrel, Rémond et Bouloiseau s'en retirèrent dès 1953 et elle fut poursuivie sous la responsabilité de Goubert ; après le dépouillement de plusieurs millions d'actes d'état civil, les résultats en sont restés inédits hormis ceux qui ont documenté la thèse de Goubert.

$8 \quad$ Les rares textes qui nous permettent de pénétrer la pratique concrète de ce laboratoire historique expérimental nous montrent les difficultés de coordination entre des chercheurs restés attachés à leurs recherches personnelles et les problèmes de maîtrise méthodique d'une documentation à la fois massive et lacunaire ${ }^{17}$.

9 Dès le début, le CRH a fonctionné comme source de financement pour des recherches d'histoire économique entreprises par des chercheurs extérieurs. Ainsi, il subventionna les enquêtes sur le trafic de Rouen de P. Jeannin (1955/56) et les recherches de M. Mollat 
dans les archives de Lille (1955/56). Mais le trait le plus marquant du CRH pendant ses années "héroïques" fut l'élan d'une équipe jeune et internationale que F. Braudel parvint à constituer autour de son enseignement à la $\mathrm{VI}^{\mathrm{e}}$ Section. Le commentaire de D. Ozanam sur les conditions de la recherche collective au CRH me semble toujours valable et pertinent :

Il s'agit donc davantage d'un travail "convergent" que d'un travail d'équipe au sens habituel du terme. Là, l'influence d'un maitre a été prépondérante dans la formation - en dehors de tout plan préconçu - d'un faisceau d'historiens orientés vers des thèmes de travaux divers, mais étroitement liés et susceptibles de renouveler notre conception du monde à cette époque ${ }^{18}$.

Dans ses réflexions autobiographiques lors de son soixantième anniversaire, R. Romano parle «des années d'extraordinaire exaltation» dans ce groupe réuni autour de F. Braudel et il évoque les noms de Chaunu, Mauro, Jeannin, Bergier, Da Silva, Godinho, Spooner, Mandich, Tucci, Vasquez da Prada ${ }^{19}$. Pierre Chaunu, en présentant le dernier tome de Séville et l'Atlantique, se sentait tenu de rendre hommage au CRH pour les moyens mis à sa disposition - machines à écrire et à calculer, lecteur de microfilms - et pour les « conversations [qu'il] a pu avoir, autrefois, dans le Centre avec des historiens tels que MM. Jean-François Bergier, Clemens Heller, Hermann Kellenbenz, Ruggiero Romano, José Gentil da Silva, Frank C. Spooner, Alberto Tenenti, Valentino Vasquez de Prada ${ }^{20}$ ». La plupart étaient des étrangers qui prolongeaient leurs séjours de recherche à Paris grâce au $\mathrm{CNRS}^{21}$. et qui trouvaient dans l'oeuvre de F. Braudel une nouvelle approche de l'histoire de leurs nations. La plupart retournèrent dans leurs pays natals pour $\mathrm{y}$ continuer les recherches commencées au CRH dont ils avaient assuré le démarrage dynamique alors que, d'un strict point de vue hexagonal, le Centre restait marginal. Il était l'un des établissements de recherche historique les plus contestés et, de ce fait, comportait encore des risques pour les jeunes historiens français en début de carrière. Cette ouverture sur l'étranger est restée un trait typique de la $\mathrm{VI}^{\mathrm{e}}$ Section, mais elle a perdu tout poids dans l'existence même de l'institution. En ce qui concerne le CRH, R. Romano et A. Tenenti sont les seuls directeurs d'études qui témoignent encore aujourd'hui de cette période « internationale ${ }^{22}$ ».

Et les résultats de ces travaux ? 0 part les publications du $\mathrm{CRH}^{23}$, la référence classique pour évaluer ces recherches reste la deuxième édition de la Méditerranée de Braudel qui enregistre attentivement le progrès de la recherche en histoire économique et sociale des $\mathrm{XVI}^{\mathrm{e}}-\mathrm{XVII}^{\mathrm{e}}$ siècles méditerranéens entre 1947 et 1964, dont les membres du CRH restaient les principaux auteurs.

\section{NOTES}

1. R. Romano, "Encore des illusions »,. op. cit., p. 16.

2. Rapport général du laboratoire de recherches historiques du 3 novembre 1949, archives EHESS, fonds Velay.

3. Micheline Baulant, Lettres de négociants marseillais : les frères Hermite (1570-1612), Paris, 1953. 
4. Vital Chomel, J.-G.. Ebersolt, Cinq siècles de circulation internationale vue de Jougne: un péage jurassien $d u \mathrm{XIII}^{\mathrm{e}}$ au XVII ${ }^{\mathrm{e}}$ siècle, avec une introduction de Lucien Febvre et un avant-propos de Fernand Braudel, Paris, 1951.

5. Rapport d'activité du Centre de recherches historiques pour l'année scolaire 1952-1953, fonds Velay, archives EHESS.

6. F. Braudel, R. Romano, Navires et marchandises à l'entrée du Port de Livourne (1547-1611), Collection Ports - Routes - Trafics, Paris, 1951, p. 7 Pour donner quelques exemples de ces publications, citons : Henri Lapeyre, Simon Ruiz et les “asientos" de Philippe II, 1953, du même, Une famille de marchands, les Ruiz. Contribution à l'étude du commerce entre la France et l'Espagne au temps de Philippe II, 1955, A. Tenenti, R. Romano, Commerce et prix du blé à Marseille au XVIII ${ }^{\mathrm{e}}$ siècle, Huguette et Pierre Chaunu, Séville et l'Atlantique, 8 vol., 1955-1960, M. Mollat, Comptabilité du port de Dieppe au $\mathrm{XV}^{\mathrm{e}}$ siècle; Trocmé et Delafosse, Le Commerce rochelais de la fin $d u \mathrm{XV}^{\mathrm{e}}$ siècle au début du XVII siècle, 1953.

7. Ainsi le premier volume de la nouvelle série «Industrie et Artisanat », J. Bouvier, F. Furet et M. Gillet, Le mouvement du profit en France au XIX siècle, Paris, La Haye 1965.

8. Une réponse à une circulaire de la Direction de l'Enseignement Supérieur concernant les taux de vacations à la VIe Section résume ainsi l'activité déployée: "Dans le cadre du Centre de Recherche d'Histoire Économique et Sociale le travail le plus fréquent est le travail de publication » (archives EHESS, fonds Velay).

9. Budget de la VIe Section de l'EPHE pour 1955, archives EHESS 66 AJ B 23.

10. Archives EHESS, fonds Velay.

11. Par exemple, Industrialisation et technocratie, sous la direction de G. Gurvitch avec une préface de L. Febvre, Paris, 1949 ; P. Francastel, L'Art mosan. Mémoires et exposés du comte J. de Borchgrave d'Alténa et autres, avec une Préface de L. Febvre, 1953; Villes et campagnes, civilisation urbaine et civilisation rurale en France, sous la direction et avec une introduction de G. Friedmann, Paris, 1953. 12. Dans la Bibliothèque Générale, L. Febvre, Au cœur religieux du XVI siècle, 1957 et Pour une histoire à part entière, 1962. dans les Cahiers des Annales; C. Morazé, Trois essais sur Histoire et Culture; M. Bloch, Apologie pour l'histoire, Esquisse d'une histoire monétaire de l'Europe, La France des derniers Capétiens, Seigneurie française et manoir anglais.

13. A. Sapori, Le marchand italien au Moyen Age, 1952 ; G. Mandich, Le pacte de "ricorsa" et le marché italien des changes au XVII siècle, 1953 ; C. M. Cipolla, Mouvements monétaires dans l'État de Milan (1580-1700), 1952 ; F. Spooner, L'économie mondiale et les frappes monétaires en France, 1956. A ces titres parus dans les trois collections classiques, il faut ajouter tous ceux de la petite collection "CEuvres étrangères »: S .Hoszowski, Les prix à Lwow (XVI ${ }^{e}$ XVII ${ }^{e}$ siècles), R. Ehrenberg, Le siècle des Fugger, 1955 ; A.G. Mankov, Le mouvement des prix dans l'État russe du XVI ${ }^{\mathrm{e}}$ siècle, 1957.

14. Par exemple, celles de Duby, Lapeyre, Vilar, Bouvier, Chaunu, Goubert et Baehrel.

15. Rapport présenté à la réunion du CRH du 19 décembre 1957, archives EHESS, fonds Velay.

16. Procès verbal d'une réunion consacrée aux travaux poursuivis par le CRH le 24 juin 1960 : "L'histoire "préfabriquée" a été accueillie avec une libéralité dont M. Braudel (qui s'en déclare responsable) est le premier à dire qu'elle fut excessive. » (archives EHESS, fonds Velay).

17. Lettres de R. Baehrel et de H. Lapeyre à F. Braudel, archives EHESS, fonds Velay.

18. D. Ozanam, Rapport pour la réunion du CRH du 12. XII. 1957, op. cit. p. 6.

19. R. Romano, op. cit., p. 17.

20. Pierre Chaunu, Séville et l'Atlantique, T. VIII.1, Paris, 1959, p. XIII.

21. Presque tous étaient stagiaires ou attachés de recherche au CNRS avec F. Braudel comme directeur de recherche ou parrain. La liste complète de tous les chercheurs du CNRS dont le directeur était F. Braudel comprend 43 noms pour la période 1950-1957. 
22. Frank C. Spooner et G. da Silva étaient les deux autres étrangers de la première heure qui gardèrent pendant longtemps des rapports réguliers et organiques, en tant que chargés de conférence et responsables d'enquêtes, avec le CRH.

23. Dans la collection «Affaires et gens d'affaires » il faut mentionner les travaux d'édition de G. da Silva, de U. Tucci, de V. Vazquez de Prada, de F. Ruiz Martin et de C. Marciani ; dans la Collection « Ports - Routes - Trafics », les livres de R. Romano et A. Tenenti ; et dans la Collection « Monnaie - Prix - Conjoncture », les ouvrages de V. Magalhaes Godinho, de R. Romano et de F.C. Spooner. 